\title{
Analgesic effects of intra-articular ropivacaine following arthroscopic knee surgery: Results of a double-blind, randomized controlled trial between two different doses
}

\author{
Rajdip Hazra', Md. Babrak Manuar ${ }^{1}$, Manjunatha S.M. ${ }^{1}$, Sisir Chakraborty ${ }^{2}$, Kaushik Ghosh ${ }^{3}$, Rajarshi \\ Basu $^{4}$, Md. Nurejjaman ${ }^{5}$, Arun Kumar N. ${ }^{6}$, Yathish S.K. ${ }^{7}$ \\ ${ }^{1}$ Department of Anesthesiology, Nilratan Sircar Medical College and Hospital, Kolkata, India, ${ }^{2}$ Department of Medicine, College of Medicine \\ and Sagore Dutta Hospital, Kolkata, India, ${ }^{3}$ Department of Medicine, Malda Medical College and Hospital, Malda, India, ${ }^{4}$ Department of \\ Pediatric Medicine, Nilratan Sircar Medical College and Hospital, Kolkata, India, ${ }^{5}$ Department of Anesthesiology, Malda Medical College and \\ Hospital, Malda, India, ${ }^{6}$ Department of Medicine, Mysore Medical College, Mysore, India, ${ }^{7}$ Department of Anesthesiology, Hindu Rao Hospital \\ and NDMC Medical College, New Delhi, India
}

\section{A B S TR A C T}

Background: Though local anesthetic agents are widely used to provide postoperative analgesia in intra-articular route, their efficacy is not accepted universally. Objective: The present study has been conducted to compare the analgesic effects of intra-articular ropivacaine between two different doses following arthroscopic knee surgery. Study design: Double-blinded randomized controlled trial. Total 60 patients of ASA (American Society of Anesthesiologists) physical status I and II, 34 male and 26 female, aged between 18 to 60 years, were randomized into 3 groups receiving $10 \mathrm{ml}$ normal saline, $10 \mathrm{ml} 0.5 \%$ ropivacaine and $10 \mathrm{ml} 0.75 \%$ ropivacaine respectively, administered in intra-articular space at the end of operation. Results: Ropivacaine groups had significantly delayed first post-operative rescue analgesic request with significantly lesser requirement of rescue analgesia in first 24 hours $(p<0.001$ in both cases). Except 6 th post-operative hours, ropivacaine groups also showed significantly less VAS scores (Visual Analogue Scale) up to 12 hours post-operatively $(p<0.05)$. Conclusion: In our double-blinded randomized controlled trial, administration of intra-articular $0.75 \%$ ropivacaine showed superior analgesic efficacy following arthroscopic knee surgery. Further, larger trials are needed to confirm our findings.

Key words: Ropivacaine, analgesia, Intra-articular route, Arthroscopic knee surgery
Access this article online Website:

http://nepjol.info/index.php/AJMS

\section{INTRODUCTION}

Arthroscopic knee surgery is one of the most common minimally invasive surgical procedures in modern orthopedic setup. A significant number of patients experience moderate to severe pain 24 hours after ambulatory surgery, particularly knee arthroscopy. ${ }^{1}$ The pain is caused by irritation of free nerve endings of the synovial tissue, anterior fat pad and joint capsule due to surgical excision and resection. ${ }^{2}$ Numerous factors have been implicated to influence post-arthroscopy pain: anesthesia technique, residual effects of perioperative analgesia, sensitivity of postoperative analgesia methods, preoperative pain level, amount of surgical trauma (i.e. diagnostic arthroscopy or arthroscopic surgery), use and duration of tourniquet exsanguinations, experience of surgeons, sex of patients and postoperative activity level of patients. ${ }^{3,4}$ But the most significant factor is postoperative time elapsed since surgery. ${ }^{5}$ In an effort to provide an effective, safe and long lasting post-arthroscopy analgesia, several studies using different drugs and regimes have been published during the last two decades. Intra-articular administration of local anesthetics has been widely used but some studies have questioned their efficacy. ${ }^{6-8}$ Use of 
subarachnoid block has been shown to negate the analgesic effects of additional local anesthetics in intra-articular route.

Recently, chondrolysis has been reported following use of amide type of local anesthetic agents in shoulder arthroscopy. ${ }^{10}$ However, single dose intra-articular injection of local anesthetic appears to be safe in the knee and ropivacaine appears to be less toxic than bupivacaine. ${ }^{11}$

The present study was designed in a prospective, randomized, double-blind fashion to compare the analgesic effects of intra-articular ropivacaine between two different doses $(10 \mathrm{ml}$ of $0.5 \%$ and $0.75 \%)$ following arthroscopic knee surgery. General anesthesia was used to eliminate possible confounding of regional anesthesia.

\section{MATERIALS AND METHODS}

After approval from institutional ethical committee and obtaining written informed consent of the patients, the study was conducted in Nilratan Sircar Medical College and Hospital, a tertiary care medical college hospital in eastern India. Total 60 patients of ASA (American Society of Anesthesiologists) physical status I and II, 34 male and 26 female, aged between 18 to 60 years and scheduled for arthroscopic knee surgery, were enrolled in this study. Power calculations suggested that a minimum of 14 subjects per group were required to detect 30 minutes difference in mean time of 1 st dose rescue analgesic requirement between groups [taking type I or $\alpha$ error of $5 \%$, type II or $\beta$ error of $20 \%$ and inter group standard deviation (SD) of 40 minutes]. To be on a safer side, 20 patients were included in each group $(n=20)$.

Patients having respiratory, cardiovascular, hepatic, renal or neurological disease; allergic to study drugs used; received analgesics for long term or within 48 hours of surgery; having body weight more than $30 \%$ of the ideal weight and those with osteoarthritis (OA) of the knee- known or discovered during surgery, were excluded from the study. Patients requiring additive analgesia intraoperatively (apart from injection fentanyl given during premedication) were also excluded from the study.

Patients were admitted one day prior to the scheduled surgery and were examined and interviewed. Whole procedure including $10 \mathrm{~cm}$ visual analogue scale $(V A S)(0$ $=$ no pain and $10=$ worst pain imaginable) was explained to the patients. On arrival in the operation theatre (OT), monitors were attached and baseline parameters like HR (heart rate), NIBP (non invasive blood pressure), $\mathrm{SpO}_{2}$ (oxygen saturation), ECG (electrocardiogram) and temperature were recorded.
Immediately before anesthesia, patients were randomly divided into three equal groups $(\mathrm{n}=20)$ using sealed envelopes chosen by the patients.

The study groups were:

i) Control group (group C) - received normal saline $(0.9 \%$ sodium chloride).

ii) Treatment groups:

a) Group R1 - received 0.5\% ropivacaine.

b) Group $\mathrm{R} 2$ - received $0.75 \%$ ropivacaine.

Total volume of the study drugs were adjusted to $10 \mathrm{ml}$ and administered intra-articularly through arthroscope after completion of surgery.

After pre-oxygenation for 3 minutes, anesthesia was induced with a standard anesthetic protocol using midazolam $0.05 \mathrm{mg} / \mathrm{kg}$, fentanyl $2 \mu \mathrm{g} / \mathrm{kg}$, thiopentone sodium 3-5 mg/kg, and tracheal intubation was facilitated by vecuronium bromide $0.1 \mathrm{mg} / \mathrm{kg}$ intravenously (IV). Anesthesia was maintained with isoflurane $(1-2 \%)$ with nitrous oxide $(66 \%)$ in oxygen. Majority of the patients had undergone repair of anterior cruciate ligament. During surgery, Ringer's lactate (RL) was administered in maintenance dose as per Holiday Segar formula.

At the end of surgery before skin closure, study drug was administered by the surgeon through port site in the intraarticular space. Tourniquet was kept inflated for another 20 minutes. Drain put by the surgeon was clamped before administering the study drug and remained clamped for another 20 minutes.

After completion of surgery, neuromuscular blockade was antagonized with injection of neostigmine $0.05 \mathrm{mg} / \mathrm{kg}$ and glycopyrrolate $0.02 \mathrm{mg} / \mathrm{kg}$ IV and patient was extubated when respiration was deemed sufficient and he/she was able to obey commands. Then patient was shifted to PACU (post anesthesia care unit).

$\mathrm{HR}, \mathrm{NIBP}, \mathrm{SpO}_{2}$, ECG and VAS scores were recorded at $1^{\text {st }}, 2^{\text {nd }}, 4^{\text {th }}, 6^{\text {th }}, 12^{\text {th }}$ and $24^{\text {th }}$ postoperative hours. Injection diclofenac sodium (75 mg IM) was given as rescue analgesic if $\mathrm{VAS}>4$. First postoperative analgesia request time, total diclofenac used in first 24 hours were recorded.

\section{Statstical analysis}

Data were expressed as mean and standard deviation (Mean $\pm \mathrm{SD}$ ). Normality of the distribution in each group was checked by the Shapiro-Wilk normality test. The homogeneity in 3 groups of mean and SD were analyzed using SPSS software version 18.0 (SPSS Inc., Chicago, Illinois, USA). Inter-group comparisons were done using one way analysis of variance (ANOVA). Post-hoc Tukey test was followed where ANOVA values were significant. Categorical data were 
compared using Chi-square test. A $p$ value of less than 0.05 was considered as statistically 'significant' $(p<0.05)$ (Figure 1).

\section{RESULTS}

There were no significant differences between the three groups with regard to demographic data such as age, sex, weight, height and duration of surgery (Table 1).

\begin{tabular}{lcccc}
\hline \multicolumn{4}{l}{ Table 1: Demographic profile (mean \pm SD) } \\
$\begin{array}{l}\text { Demographic } \\
\text { profile }\end{array}$ & Group C & Group R1 & Group R2 & p value \\
\hline Age (years) & $36.4 \pm 9.65$ & $33.15 \pm 10$ & $32.15 \pm 8.88$ & 0.343 \\
Sex (M:F) & $12: 8$ & $9: 11$ & $13: 7$ & 0.415 \\
Weight (Kg) & $63.1 \pm 8.54$ & $65.25 \pm 9.59$ & $61.6 \pm 9.9$ & 0.469 \\
Height (cm) & $161.6 \pm 9.66$ & $163.8 \pm 9.67$ & $162.55 \pm 6.95$ & 0.734 \\
$\begin{array}{l}\text { Duration of } \\
\text { surgery (min) }\end{array}$ & $110.2 \pm 17.9$ & $107.15 \pm 12.73$ & $109.65 \pm 14.61$ & 0.797 \\
\hline
\end{tabular}

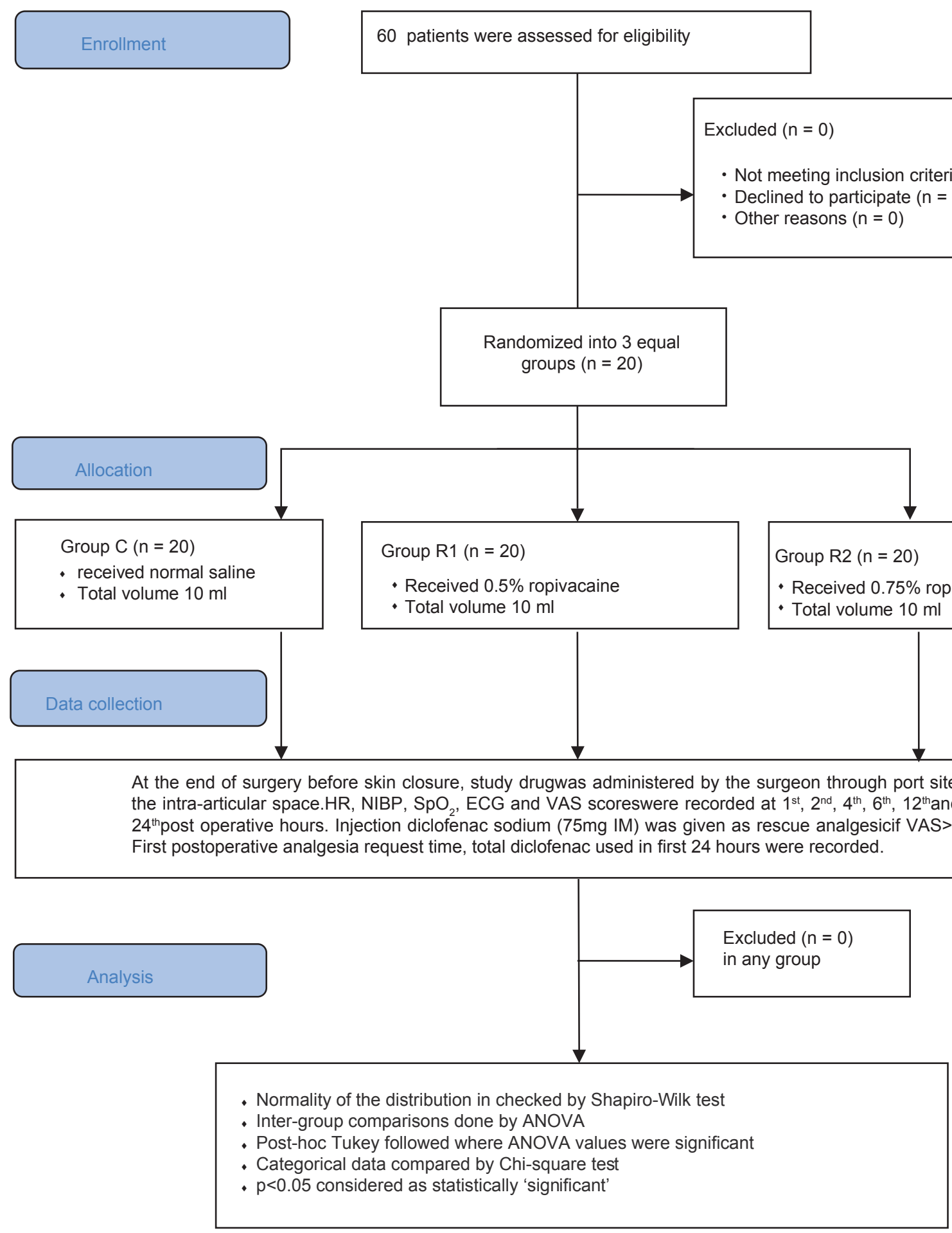

Figure 1: The CONSORT flow diagram of the study design 
Comparison of time of first postoperative rescue analgesic request $\left(\mathrm{RA}_{1}\right)$ and total number of rescue analgesic requirement in first 24 hours postoperatively $\left(\mathrm{RA}_{\mathrm{T}}\right)$ are shown in Table 2. Compared to the control group, ropivacaine groups (both R1 and R2) had significantly delayed first post-operative rescue analgesic request with significantly lesser requirement of rescue analgesia in first 24 hours ( $x<0.001$ in both cases). Between group R1 and R2, though $\mathrm{RA}_{1}$ was significantly different, there was no significant difference in $\mathrm{RA}_{T}$, values.

Figure 2 shows the VAS scores of the three groups at $1^{\text {st }}$, $2^{\text {nd }}, 4^{\text {th }}, 6^{\text {th }}, 12^{\text {th }}$ and $24^{\text {th }}$ postoperative hours. The VAS score was 0 in all the groups at 1 st post operative hour. Except 6th postoperative hours, VAS scores were significantly lower in ropivacaine groups (both R1 and R2) all the time $(p<0.05)$. However, at 24 th postoperative hours, no significant difference in VAS scores between the three groups was observed $(p>0.05)$.

No incidence of adverse events (AEs) like nausea, vomiting, urinary retention, itching or sedation was observed in any study group.

Table 2: Time of first rescue analgesic and total
no. of rescue analgesic in first 24 hrs (mean $\pm S D)$

$\mathrm{RA}_{1}=$ time of first post-operative rescue analgesic request (in minutes); $\mathrm{RA}_{\mathrm{T}}=$ total number of rescue analgesic requirement in first 24 hours post-operatively. Symbols represent a significant difference $(p<0.05)$ compared with the control group $C\left({ }^{*}\right)$ or between group $\mathrm{R}_{1}$ and $\mathrm{R}_{2}(\dagger)$, as determined using a one-way ANOVA with a post-hoc Tukey test

\section{DISCUSSION}

Arthroscopic surgery is associated with a variable amount of postoperative pain. ${ }^{1}$ Many modes of postoperative analgesia have been reported for patients undergoing knee arthroscopy. It can be provided by systemic administration of either narcotic or non-narcotic analgesic drugs; ${ }^{12}$ intra-articular local anesthetic drugs, ${ }^{6}$ non-narcotic analgesic drugs (ketorolac) ${ }^{13}$ or narcotic analgesic drugs (morphine, pethidine and fentanyl). ${ }^{14,15}$ Each route has its own advantages and disadvantages. Intermittent systemic analgesic administration can not keep the patient totally pain free for all the times and also often associated with side effects like nausea, vomiting, respiratory depression, drowsiness, pruritus, reduced gut motility and urinary retention; ${ }^{16}$ whereas intra-articular route provides qualitatively better analgesia locally with minimal systemic side effects. It may be due to slower rate of absorption through poorly vascular intra-articular surface. Intra-articular drug administration is one of the simplest techniques requiring no specialized equipment for pain management, but it is applicable only during surgery.

In last two decades, there have been various studies using different drugs via intra-articular route for postoperative pain management after athroscopy. Geutens et al. demonstrated the analgesic effect of $0.5 \%$ intra-articular bupivacaine in first post-operative day. ${ }^{17}$ Ropivacaine is a commonly used local anesthetic agent and is structurally related to bupivacaine. It causes reversible inhibition of sodium influx during action potential generation thus blocking impulse conduction in nerve fibers. This action is also potentiated by dose dependent inhibition of potassium

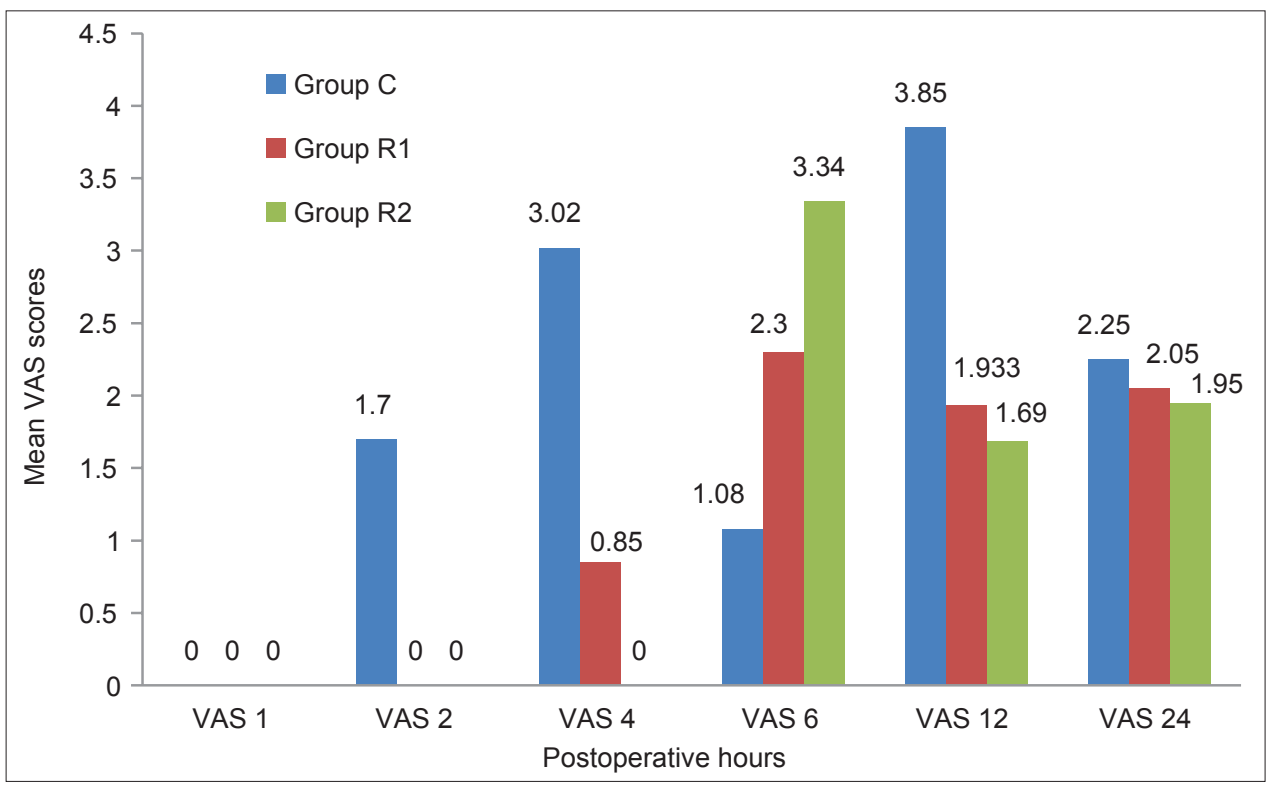

Figure 2: Mean VAS scores of study groups at different post-operative hours 
channels. ${ }^{18}$ Ropivacaine is available as an optically pure $\mathrm{S}(-)$ enantiomer from the parent chiral molecule propivacaine, ${ }^{19}$ whereas bupivacaine is a mixture of two enantiomers- $\mathrm{R}(+)$ and $\mathrm{S}(-) . \mathrm{R}(+)$ enantiomer is responsible for the toxicity of the local anesthetic agent. Ropivacaine is less lipid soluble than bupivacaine, but its pharmacokinetic deposition is similar. Due to decreased lipophilicity, it is less likely to penetrate large myelinated $A \beta$ motor fibers than paintransmitting $A \delta$ and $C$ fibers. For these reasons, ropivacaine seems to provide similar and effective post-arthroscopy analgesia compared to bupivacaine with less CNS (Central nervous system) and cardiac toxicity. ${ }^{20}$ Convery et al. studied plasma concentrations of ropivacaine following single intra-articular injection in the knee joint and found that single dose ropivacaine up to $200 \mathrm{mg}$ cut down below the estimated threshold level for systemic toxicity. ${ }^{21}$ They also observed that median analgesic consumption in the first and second postoperative 24 hour periods were lower in the ropivacaine groups than bupivacaine group. Samoladas and colleaguesconcluded that intra-articular ropivacaine is effective to reduce postoperative pain minimizing the use of systemic analgesia. ${ }^{22}$ Mar ret $e t$ al. concluded that intra-articular ropivacaine $0.75 \%$ provides better analgesia than bupivacaine $0.5 \%$ (no significant differences in VAS scores compared to control with bupivacaine $0.5 \%$ ) after arthroscopic knee surgery. ${ }^{23}$ Chen et al. established the safety and efficacy of intra-articular ropivacaine in Chinese population. ${ }^{24}$ Franceschi et al. compared $75 \mathrm{mg}$ ropivacaine and $2 \mathrm{mg}$ morphine intra-articularly and showed that ropivacaine is a safe, site-specific, and long lasting anesthetic drug with an earlier onset of action than morphine and almost the same duration during the entire postoperative period. ${ }^{16}$

In this study, ropivacaine group had significantly delayed first post-operative rescue analgesic request with significantly lesser requirement of rescue analgesia in first 24 hours ( $p<$ 0.001 in both cases). Except 6th and 24th post-operative hours, ropivacaine group patients also showed significantly less VAS scores up to 24 hours post-operatively $(p<0.05)$. The higher mean VAS score at 6 th postoperative hours in ropivacaine groups (both R1 and R2) were probably due to administration of rescue analgesics in other groups before the time of scheduled observation. At 24th postoperative hours, there was no significant difference in VAS scores between control and ropivacaine groups, both R1 and R2 $(p>0.05)$. So, it was clearly found that analgesic efficacy of intra-articular ropivacaine is significantly $(p<0.05)$ superior than placebo, at least up to first 12 hours postoperatively.

The results of our study support the findings of Samoladas et al..$^{22}$ The comparatively lower VAS scores in the ropivacaine groups may be due to the fact that use of tourniquet and rescues analgesics may modify VAS scores.
Significant lower VAS score as observed by Convery et al. is probably due to comparatively higher doses of ropivacaine and usage of additional lignocaine for port site infiltration. ${ }^{21}$ We also support the findings of Marret et al. ${ }^{23}$ Chen et al. ${ }^{24}$ and Franceschi et al. ${ }^{16}$

We contradict the findings of Aasbø et al. ${ }^{7}$ and Rautoma et $a l^{8}$ regarding the inefficacy of intra-articular local anesthetic agents. This is probably due to use of low concentrations of local anesthetics $(0.25 \% \text { bupivacaine })^{7}$ or use of subarachnoid block ${ }^{8}$ which may negate the analgesic effects of additional local anesthetics in intra-articular route. ${ }^{9}$ After meta-analysis of twenty double blinded randomized controlled trials, Møiniche et al. concluded that intra-articular local anesthetic agents may have moderate analgesic activity of short duration. ${ }^{6}$ These findings are in support of our study.

Recently, chondrolysis has been reported following intraarticular use of amide type of local anesthetic agents. ${ }^{10}$ It is a separate entity from that of systemic toxicity. Chondrolysis has been defined as the destruction of articular cartilage due to dissolution of cartilage matrix and chondrocytes over a very short period of time. ${ }^{10} \mathrm{It}$ can lead to severe OA and long-term disability in future. Though local anesthetics have been implicated as one of the causative agents of chondrolysis, it is associated with prolonged infusion at high doses. Until further data are available, single intra-articular injection of local anesthetic agents may be taken as safe. Young and Buvanendran opined that local anesthetics are probably safe for intra-articular use in the knee. ${ }^{25}$ However, they have discouraged continuous and patient-controlled intraarticular infusions.

There were several limitations of this study like small sample size, single-center design and selected study population (pediatric, elderly and ASA III-IV patients were excluded). Also there was no follow up to detect any local tissue damage in intra-articular space following injection of study drugs. Further large scale multicentric clinical studies can be performed to develop a more reliable and clinically efficient regime of ropivacaine as a component of multimodal approach of post operative analgesia without any side effects.

\section{CONCLUSION}

In our double-blind randomized controlled trial, administration of intra-articular ropivacaine showed superior analgesic efficacy when compared with placebo following arthroscopic knee surgery. We recommend routine use of $0.75 \%$ ropivacaine in intra-articular route 
following arthroscopic knee surgery. Further, larger trials are needed to confirm our findings and better delineate the clinically efficient regime of ropivacaine.

\section{ACKNOWLEDGEMENTS}

The authors like to acknowledge Nilratan Sircar Medical College and Hospital, Kolkata, India for providing necessary support during this research work. None of the authors have any conflict of interest.

\section{REFERENCES}

1. Pavlin DJ, Chen C, Penaloza DA and Buckley FP. A survey of pain and other symptoms that affect the recovery process after discharge from an ambulatory surgery unit. J Clin Anesth 2004; 16(3):200-206.

2. Dye SF, Vaupel GL and Dye CC. Conscious neurosensory mapping of the internal structures of the human knee without intraarticular anesthesia. Am J Sports Med 1998; 26(6):773-777.

3. Kirkley A, Rampersaud R, Griffin S, Amendola A, Litchfield R and Fowler $P$. Tourniquet versus no tourniquet use in routine knee arthroscopy: a prospective, double-blind, randomized clinical trial. Arthroscopy 2000; 16(2):121-126.

4. Rosseland LA and Stubhaug A. Gender is a confounding factor in pain trials: women report more pain than men after arthroscopic surgery. Pain 2004; 112(3):248-253.

5. Sutariya B, Patel A, Desai A and Shaikh S. Post-Operative Pain after Knee Arthroscopy and Related Factors. Natl J Med Res 2014; 4(1):75-78.

6. Møiniche S, Mikkelsen S, Wetterslev J and Dahl JB. A systematic review of intra-articular local anesthesia for postoperative pain relief after arthroscopic knee surgery. Reg Anesth Pain Med 1999; 24(5):430-437.

7. Aasbø V, Raeder JC, Grøgaard B and Røise O. No additional analgesic effect of intra-articular morphine or bupivacaine compared with placebo after elective knee arthroscopy. Acta Anaesthesiol Scand 1996; 40(5):585-588.

8. Rautoma P, Santanen U, Avela R, Luurila H, Perhoniemi V and Erkola O. Diclofenac premedication but not intra-articular ropivacaine alleviates pain following day-case knee arthroscopy. Can J Anaesth 2000; 47(3):220-224.

9. Santanen U, Rautoma P, Luurila H and Erkola O. Intra-articular ropivacaine injection does not alleviate pain after day-case knee arthroscopy performed under spinal anaesthesia. Ann Chir Gynaecol 2001; 90(1):47-50.

10. Bailie DS and Ellenbecker TS. Severe chondrolysis after shoulder arthroscopy: a case series. J Shoulder Elbow Surg 2009; 18(5):742-747.
11. Webb ST and Ghosh S. Intra-articular bupivacaine: potentially chondrotoxic? Br J Anaesth 2009; 102(4):439-441.

12. Gürkan $Y$, Kiliçkan L, Buluc L, Müezzinoglu $S$ and Toker $K$. Effects of diclofenac and intra-articular morphine/bupivacaine on postarthroscopic pain control. Minerva Anestesiol 1999; 65(10):741-745.

13. Gupta A, Axelsson K, Allvin R, Liszka-Hackzell J, Rawal N, Althoff $B$, et al. Postoperative pain following knee arthroscopy: the effects of intra-articular ketorolac and/or morphine. Reg Anesth Pain Med 1999; 24(3):225-230.

14. Varkel V, Volpin G, Ben-David B, Said R, Grimberg B, Simon K, et al. Intra-articular fentanyl compared with morphine for pain relief following arthroscopic knee surgery. Can J Anaesth 1999; 46(9):867-871.

15. Söderlund A, Westman L, Ersmark H, Eriksson E, Valentin A and Ekblom A. Analgesia following arthroscopy- a comparison of intra-articular morphine, pethidine and fentanyl. Acta Anaesthesiol Scand 1997; 41(1):6-11.

16. Franceschi F, Rizzello G, Cataldo R and Denaro V. Comparison of morphine and ropivacaine following knee arthroscopy. Arthroscopy 2001; 17(5):477-480.

17. Geutens $G$ and Hambridge JE. Analgesic effects of intraarticular bupivacaine after day-case arthroscopy. Arthroscopy 1994; 10(3):299-300.

18. Kindler CH, Paul M, Zou H, Liu C, Winegar BD, Gray AT and Yost CS. Amide local anesthetics potently inhibit the human tandem pore domain background $\mathrm{K}+$ channel TASK-2 (KCNK5). J Pharmacol Exp Ther. 2003; 306(1):84-92.

19. Kuthiala $G$ and Chaudhary G. Ropivacaine: A review of its pharmacology and clinical use. Indian J Anaesth 2011; 55(2):104-110.

20. Scott DB, Lee A, Fagan D, Bowler GM, Bloomfield P and Lundh R. Acute toxicity of ropivacaine compared with that of bupivacaine. Anesth Analg 1989; 69(5):563-569.

21. Convery PN, Milligan KR, Quinn P, Sjövall J and Gustafsson U. Efficacy and uptake of ropivacaine and bupivacaine after single intra-articular injection in the knee joint. $\mathrm{Br} \mathrm{J}$ Anaesth 2001; 87(4):570-576.

22. Samoladas EP, Chalidis B, Fotiadis H, Terzidis I, Ntobas T and Koimtzis M. The intra-articular use of ropivacaine for the control of post knee arthroscopy pain. J Orthop Surg Res 2006; 23:1:17.

23. Marret E, Gentili M, Bonnet MP and Bonnet F. Intra-articular ropivacaine $0.75 \%$ and bupivacaine $0.50 \%$ for analgesia after arthroscopic knee surgery: a randomized prospective study. Arthroscopy 2005; 21(3):313-316.

24. Chen S, Chen Z, Jin Y, Tian Z, Lin X, Zhu M, et al. Pharmacokinetics and efficacy of ropivacaine in Chinese patients following intra-articular administration. Int J Clin Pharmacol Ther 2013; 51(5):393-400.

25. Young $A$ and Buvanendran A. Multimodal systemic and intra-articular analgesics. Int Anesthesiol Clin. 2011; 49(4):117-133.

\section{Authors Contribution:}

RH \& MBM - Plan of study; RH, MSM, RB \& MN - Preparation of the initial draft; KG, AKN, YSK - Contribution to the manuscript; RH \& SC - Statistical analysis; RH - Review and final preparation of the manuscript

Source of Support: Nil, Conflict of Interest: None declared. 